\title{
Arthritis charter sets patient duty to engage
}

$\mathrm{T}$ he Canadian Arthritis Patient Alliance (CAPA) is urging those with the disease to take charge of their care with a new charter of rights and responsibilities.

The Arthritis Patient Charter states that patients living with the disease have a responsibility to learn about their condition and its management, live a healthy lifestyle, speak openly with their health care providers and follow agreed-upon treatments. It also affirms rights to timely diagnosis, equal reimbursement and access to medication, and participation in treatment decisions.

Despite recent strides in treatment for the 100-plus muscle and joint conditions that fall under the banner of arthritis, these conditions are still underdiagnosed and undermanaged in Canada, says CAPA President Linda Wilhelm.

Part of the problem is a misconception that arthritis is just "minor aches and pains that come with getting older," she says. In fact, two-thirds of Canada's 4.6 million patients with arthritis are under 65. Many have inflammation that can cause permanent joint damage and crippling pain without immediate treatment.

Time is of the essence when it comes to finding the right treatment, says Laurie Proulx, a member of CAPA's steering committee, in whom juvenile idiopathic arthritis was diagnosed at age 14 . "People can start to show joint damage in less than two months," says Proulx. Yet, "they sometimes wait years for a diagnosis or appropriate treatment for a multitude of reasons." These include late referral to a rheumatologist for more accurate diagnosis, and physician reluctance to prescribe expensive new treatments without exhausting other options.

Access to biologic response modifiers - the standard of care worldwide for treatment of severe rheumatoid arthritis — is particularly poor in Canada, says Wilhelm. The majority of biologics are available only on a case-by-case basis hrough a lengthy review process that varies across the country. Some rheumatologists are hesitant to prescribe the

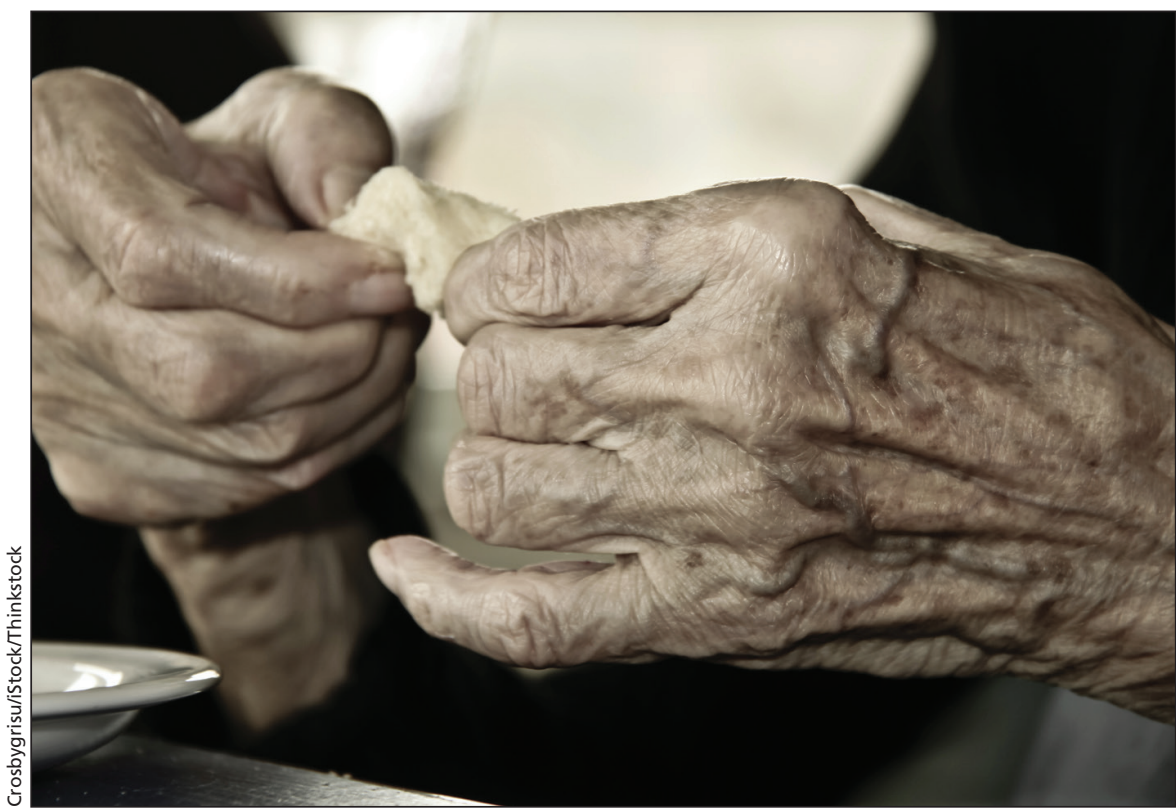

Many Canadians, including physicians and patients with arthritis, underestimate the seriousness of disease, say patient advocates.

drugs for fear of "backlash from insurance companies and public formularies because of the cost," explains Wilhelm. Others "still perceive biologics [which first came into use in the late 1990s] as somewhat new.'

Meanwhile, the arsenal of treatments for arthritis pain is "practically nonexistent," as a result of government crackdowns on opioid use for noncancer pain and new professional guidelines that discourage prescribing cannabis to people with arthritis.

In such an environment, patients can't afford to be passive, says Wilhelm. "If you're not involved in your care, you're going to have poor outcomes."

For example, few patients get serious about lifestyle changes until they're already in "big trouble," she says. Although weight control and exercise are important to managing arthritis, the Public Health Agency of Canada reports that patients with the disease are more likely to be inactive in their leisure time, overweight or obese than people who don't have arthritis.

Patient education and advocacy are essential to better outcomes, says
Proulx. She experienced first hand the difference a "good advocate" can make. At age 21, and in extreme pain, "I was told there wasn't much else they could prescribe," she recounts. Proulx's father researched further, and learning about biologics, pushed for her to receive treatment in the United States until the drugs were approved in Canada - an option her physician hadn't considered.

"You've got to take action for yourself because no one is going to do it for you," says Proulx.

Wilhelm acknowledges this represents a "big culture change" for most patients. "We've had pushback from patients who want the system and their doctors to fix everything," she says. "Everyone wants a quick fix ... but arthritis is a lifetime disease you have to learn to live with."

CAPA distributed the charter to rheumatologists across Canada in September. The alliance is also planning a rollout to pharmacists, physiotherapists and other health care providers next year. Lauren Vogel, CMAJ

CMAJ 2014. DOI:10.1503/cmaj.109-4922 